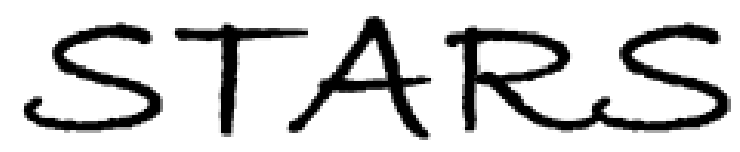

University of Central Florida

STARS

$1-1-2006$

\title{
Infrared image scintillation: comparison of modeling and measurement
}

Ingmar G. E. Renhorn

Thomas Svensson

Göran Carlsson

Staffan Cronström

Glenn D. Boreman

University of Central Florida

Find similar works at: https://stars.library.ucf.edu/facultybib2000

University of Central Florida Libraries http://library.ucf.edu

This Article is brought to you for free and open access by the Faculty Bibliography at STARS. It has been accepted for inclusion in Faculty Bibliography 2000s by an authorized administrator of STARS. For more information, please contactSTARS@ucf.edu.

\section{Recommended Citation}

Renhorn, Ingmar G. E.; Svensson, Thomas; Carlsson, Göran; Cronström, Staffan; and Boreman, Glenn D., "Infrared image scintillation: comparison of modeling and measurement" (2006). Faculty Bibliography 2000s. 6502.

https://stars.library.ucf.edu/facultybib2000/6502

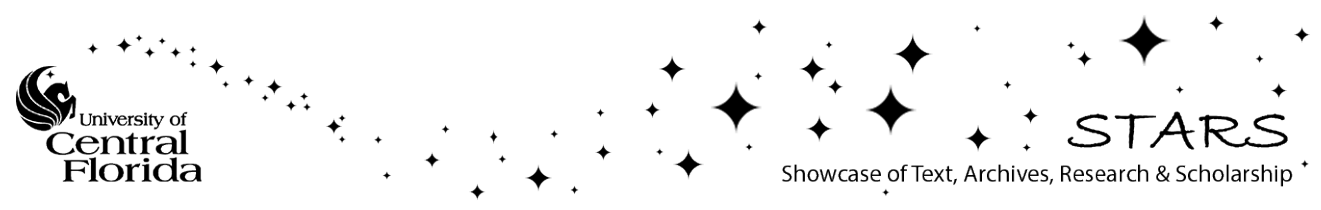




\section{Infrared image scintillation: comparison of modeling and measurement}

\author{
Ingmar G. E. Renhorn, MEMBER SPIE \\ Thomas Svensson, MEMBER SPIE \\ Göran Carlsson \\ Staffan Cronström \\ FOI-Swedish Defence Research Agency \\ P.O. Box 1165 \\ SE-581 11 Linköping \\ Sweden
}

\author{
Glenn D. Boreman, FELLOW SPIE \\ University of Central Florida \\ Center for Research and Education in \\ Optics and Lasers \\ College of Optics and Photonics \\ Orlando, Florida 32816
}

\begin{abstract}
Atmospheric scintillation effects on point targets and small targets are studied in the middle-wave infrared (MWIR) band over a 2.5-km horizontal path. Expressions are presented for both exact numerical calculations and for a Gaussian-aperture approximation of scintillation that has an accurate analytic solution. Experimental results show that measurements of $C_{n}^{2}$ using a commercial NIR scintillometer allow the prediction of average scintillation levels in the MWIR when corrections are made for wavelength and dimensions of the source and receiver. The measured log-irradiance values are approximately lognormal, and are about a factor of 2 higher than would have been expected from theory. Our results are relevant for analysis of detection and falsealarm probabilities over long horizontal paths in the MWIR. ๑ 2006 Society of Photo-Optical Instrumentation Engineers. [DOI: 10.1117/1.2158976]
\end{abstract}

Subject terms: middle-wave infrared; image scintillation; infrared; turbulence; infrared imaging.

Paper 040349RR received Jun. 4, 2004; revised manuscript received Jun. 3, 2005; accepted for publication Jun. 7, 2005; published online Jan. 24, 2006.

\section{Introduction}

Scintillation is a common phenomenon in long-path propagation through turbulent atmosphere, and has been extensively treated in the literature. ${ }^{1}$ When detecting small targets at large ranges, the signal statistics will be influenced not only by the sensor noise, the source, and background variability, but also the scintillation introduced by the atmospheric turbulence. Since many systems operate in the middle-wave infrared (MWIR) and long-wave infrared (LWIR) regions, it is important to be able to understand the source and aperture averaging phenomena and wavelength dependency. Modern scintillometers operate with sources and apertures larger than the Fresnel zone size, which makes the normalized variance for weak refractive turbulence approximately proportional to a path-averaged $C_{n}^{2}$. Forward-looking infrared (FLIR) systems operate often in the sub-Fresnel zone size, and a more detailed model describing both the wavelength dependency and the source and receiver size dependency is needed to understand the observed fluctuations. Turbulence is created when hot and cold air is mixed. The detailed interaction between meteorology and turbulence is not of key importance in this work, although such models are of interest for estimating sensor performance from macroscale weather parameters. The magnitude of the turbulence is governed by the energy budget, i.e., the energy exchange between the air and the ground, and by the skewing of the air stream due to friction. During daytime, when the present experiments were performed, typically large-scale turbulent eddies are observed when the ground is heated by solar radiation. The ground heats the lowest layer of air, causing air bubbles to rise (unstable atmosphere)., Wind mixes these bubbles into a turbulent mixture, which influences the optical

0091-3286/2006/\$22.00 @ 2006 SPIE propagation as an ensemble of random lenses. The smallest bubbles will scatter by diffraction, while the larger ones will scatter by refraction. During dawn and dusk, a neutral atmosphere with very low turbulence can develop. During clear nights, the ground cools off and a stable situation can appear where the air temperature is higher than the ground temperature. This situation will also give rise to turbulence. The infrared (IR) scintillation problem is somewhat different from the corresponding phenomenon in the visible band, since IR imaging has a smaller fractional bandwidth and is thus more coherent than a full-bandwidth visible imager. The diffractive portion of the scintillation is more pronounced in the IR for a given size eddy, while the refractive portion of the scintillation is wavelength independent. The finite size of typical practical receiver apertures indicates that the effects of the inner scale are most often averaged out. However, for smaller receiver apertures than the ones considered here, there may still be significant innerscale effects on the scintillation data.

This work seeks to develop analytical approximations to the general theory appropriate for the case of image scintillation observed in the infrared band. We compare our approximations to numerical solutions of the exact expressions, as well as to scintillation data observed over a $2.5-\mathrm{km}$ path in the MWIR portion of the spectrum (three bands covering the range 2.0 to $5.2 \mu \mathrm{m}$ ) and to nearsimultaneous data obtained from a commercial nearinfrared (NIR) scintillometer operating at $0.88 \mu \mathrm{m}$. The MWIR data are obtained from thermal sources of two sizes, one essentially a point source and one an extended source. Experimental results show that measurements of $C_{n}^{2}$ using a commercial NIR scintillometer allow prediction of average scintillation levels in the MWIR when corrections are made for wavelength and dimensions of the source and receiver. The measured log-irradiance values are approximately log- 
normal, and were about a factor of 2 higher than would have been expected from theory when saturation effects are included.

Our treatment is significant to the problem of describing horizontal-path scintillation phenomenon, especially for the case of IR point-source detection. Most predictive models ${ }^{4}$ for detection range assume that the intensity of the point source is constant and develop probability of detection versus range predictions on the basis of Gaussian noise in the sensor system. The fact that the target strength itself varies, and varies lognormally, would be a useful addition to the calculations of the range models.

\section{Theoretical Background}

Approximations are needed to describe the whole range from weak to strong turbulence. Most theories ${ }^{1}$ start out from the Kolmogorov model and the corresponding 3-D power spectrum for the index of refraction fluctuations. Modifications to this spectrum have been suggested by von Karman, Tatarskii, and Hill. Andrews ${ }^{5}$ has suggested an analytical approximation to the Hill spectrum. It will be useful to have a brief definition of terms before proceeding to the development of the modified aperture-correction and path-weighting functions.

Small-scale fluctuations are associated with the first Fresnel range $R_{f}=\sqrt{L / k}$ or coherence radius $\rho_{0}$, depending on which is smallest. The variable $L$ represents the propagation range, $k$ is the wave number where $k=2 \pi / \lambda$ and $\lambda$ is the wavelength, and $z$ is the path-position variable that is 0 at the transmitter and $L$ at the receiver. Large-scale fluctuations are created by turbulence cells larger than the Fresnel range or the scattering disk $L /\left(k \rho_{0}\right)$, depending on which one is largest. The scattering disk is defined by the refractive cell of size $l$ for which the focusing angle $\theta_{F} \sim l / L$ is equal to the average scattering angle $\theta_{D} \sim 1 / k \rho_{0}$.

The most effective scale sizes for causing intensity fluctuations are for refractive effects of the Fresnel zone size at weak turbulence and scattering disk size at strong turbulence. For diffractive effects, the most effective scale size is the Fresnel zone size at weak turbulence, and spatial coherence radius $\rho_{0}$ at strong turbulence.

The spatial coherence is decreasing when propagating through a turbulent medium. The coherence radius can be obtained from the modulus of the complex degree of coherence $^{6}$ (DOC)

$\operatorname{DOC}\left(r_{1}, r_{2}, L\right)=\exp \left[-\frac{1}{2} D\left(r_{1}, r_{2}, L\right)\right]$,

where $D\left(r_{1}, r_{2}, L\right)$ is a sum of structure functions often dominated by the phase structure function. The transverse coherence radius is obtained by setting $\rho_{0}=r_{1}=-r_{2}$ and solving $D\left(\rho_{0}, L\right)=1$, i.e., for the $1 / e^{1 / 2}$ value. Assuming weak turbulence and a spherical wave front, the solution ${ }^{6}$ is

$\rho_{0}=\left(1.09 C_{n}^{2} k^{2} L l_{0}^{-1 / 3}\right)^{-1 / 2}, \quad \rho_{0} \ll l_{0}$

$\rho_{0}=\left(1.09 C_{n}^{2} k^{2} L\right)^{-3 / 5}, \quad l_{0} \ll \rho_{0} \ll L_{0}$,

where $l_{0}$ is the inner scale and $L_{0}$ is the outer scale. A small value of $\rho_{0}$ implies $^{6}$ a large scattering disk, which for our conditions of long propagation path is the dominant contribution to scintillation.

Three statistical parameters are of importance for the description of scintillation, namely, the normalized irradiance variance or "scintillation index"

$\sigma_{I}^{2}=\frac{\left\langle(I-\langle I\rangle)^{2}\right\rangle}{\langle I\rangle^{2}}=\frac{\left\langle I^{2}\right\rangle}{\langle I\rangle^{2}}-1 ;$

the mean of the logarithm of irradiance $\langle\ln I\rangle$; and the variance of the logarithm of irradiance

$\sigma_{\ln I}^{2}\left\langle(\ln I-\langle\ln I\rangle)^{2}\right\rangle$.

The log-irradiance variance is independent of scale (assume $S$ a constant scale factor), since $\ln (S I)-\langle\ln (S I)\rangle=\ln (L)$ $-\langle\ln (I)\rangle$.

The irradiance variance at weak turbulence is given by the Rytov variance $\beta_{0}^{2}$. For weak turbulence $\left(\sigma_{I}^{2} \ll 1\right)$, we have ${ }^{6}$

$\beta_{0}^{2} \equiv \sigma_{I}^{2}=\exp \left\{\sigma_{\ln I}^{2}\right\}-1 \approx \sigma_{\ln I}^{2}=-2\left\langle\ln \frac{I}{\langle I\rangle}\right\rangle$,

where the last step is simply the result of $\ln I$ obeying a lognormal distribution, for which the normalization is

$\left\langle\ln \frac{I}{\langle I\rangle}\right\rangle=-\frac{\sigma_{\ln I}^{2}}{2}$.

For isotropic turbulence, the 1-D power spectrum for the index of refraction fluctuation is given by

$\Phi_{n}(\kappa)=0.033 C_{n}^{2} \kappa^{-11 / 3} f\left(\kappa l_{0}\right), \quad 0 \leqslant \kappa<\infty$,

where $\kappa$ is the spatial wave number, and $f\left(\kappa l_{0}\right)$ is a dimensionless function that describes the spectral variation in the high wave number dissipation region. This function is normalized such that $f(0)=1$.

There are several models for the power spectrum with respect to inner and outer scale. For example, the von Karman model gives (within $0 \leqslant \kappa<\infty$ )

$\Phi_{n}(\kappa)=0.033 C_{n}^{2} \frac{\exp \left(-\kappa^{2} / \kappa_{m}^{2}\right)}{\left(\kappa^{2}+\kappa_{o}^{2}\right)^{11 / 6}}$,

where $\kappa_{m}=5.92 / l_{0}$ and $\kappa_{0}=1 / L_{0}$. The influence of the outer scale can often be disregarded by setting $\kappa_{0}=0$ in the von Karman model, which is commonly called the Tatarskii spectrum. $<\infty)$

The modified ${ }^{5}$ Hill spectrum is given by (within $0 \leqslant \kappa$

$$
\begin{aligned}
\Phi_{n}(\kappa)= & 0.033 C_{n}^{2}\left[1+1.802\left(\kappa / \kappa_{l}\right)\right. \\
& \left.-0.254\left(\kappa / \kappa_{l}\right)^{7 / 6}\right] \frac{\exp \left(-\kappa^{2} / \kappa_{l}^{2}\right)}{\left(\kappa^{2}+\kappa_{0}^{2}\right)^{11 / 6}},
\end{aligned}
$$

where $\kappa_{l}=3.3 / l_{0}$.

The log-irradiance variance can be obtained from an integral of the path-weighting function $(\mathrm{PWF})^{7}$ 
$\sigma_{\ln I}^{2}=\int_{0}^{1} d u \operatorname{PWF}(u)$

where $u$ is a dimensionless variable of path distance normalized by the range $u=z / L$. The PWF to be used depends on the assumed refractive-index spectra and on whether effects of limiting apertures are included. For sphericalwave calculations

$\mathrm{PWF}=16 \pi^{2} k^{2} L \int_{0}^{\infty} d \kappa \kappa \Phi_{n}(\kappa) \sin ^{2}\left[\frac{\kappa^{2} L u(1-u)}{2 k}\right] H_{S T}$,

$\sigma_{\ln I}^{2}=16 \pi^{2} k^{2} L \int_{0}^{1} d u \int_{0}^{\infty} d \kappa \kappa \Phi_{n}(\kappa) \sin ^{2}\left[\frac{\kappa^{2} L u(1-u)}{2 k}\right] H_{S T}$

The term $H_{S T}$ is a saturation parameter ${ }^{8,9}$ that for weak turbulence can be set to 1 . For stronger turbulence, an in- dication of saturation behavior can be obtained by evaluating $H_{S T}$ in the limits of large and small coherence radius ${ }^{8}$

$H_{S T}=\exp \left\{-\left[\frac{R_{f}^{2}}{\rho_{0}} \kappa u(1-u)\right]^{2}\right\}, \quad \rho_{0} \ll l_{0}$,

$H_{S T}=\exp \left\{-\left[\frac{R_{f}^{2}}{\rho_{0}} \kappa u(1-u)\right]^{5 / 3}\right\}, \quad \rho_{0} \gg l_{0}$,

where $R_{f}^{2} / \rho_{0}$ is the scattering disk previously defined.

In the case of a power spectrum described by $f\left(\kappa l_{0}\right)=1$ and $H_{S T}=1$, we obtain $\sigma_{\ln I}^{2}=\beta_{0}^{2}$, where the parameter $\beta_{0}^{2}$ is the Rytov variance at weak turbulence with inner scale $l_{0}$ $=0$,

$\beta_{0}^{2}=0.496 k^{7 / 6} L^{11 / 6} C_{n}^{2}$

If the inner scale is included by using the von Karman spectrum and the outer scale is neglected, the von Karman variance $\beta_{K}^{2}$ is described by

$\beta_{K}^{2}=5.211 C_{n}^{2} k^{2} L \int_{0}^{1} d u \frac{3\left[\left[1+\frac{\kappa_{l}^{4} L^{2}(1-u)^{2} u^{2}}{k^{2}}\right]^{5 / 12} \cos \left(\frac{5}{6} \arctan \left\{\kappa_{l}^{2}\left[\frac{L^{2}(1-u)^{2} u^{2}}{k^{2}}\right]^{1 / 2}\right\}\right) 1-\right] \Gamma\left[\frac{1}{6}\right]}{10 \kappa_{l}^{5 / 3}}$.

When the effects of finite transmitter and receiver areas are included, the path-weighting function is given by ${ }^{10}$

$$
\begin{aligned}
\operatorname{PWF}(u)= & 16 \pi^{2} k^{2} L \int_{0}^{\infty} d \kappa \kappa \Phi_{n}(\kappa) \sin ^{2}\left[\frac{\kappa^{2} L u(1-u)}{2 k}\right] \\
& \times\left\{\frac{2 J_{1}\left[\kappa r_{v} u\right]}{\kappa r_{v} u} \frac{2 J_{1}\left[\kappa r_{t}(1-u)\right]}{\kappa r_{t}(1-u)}\right\}^{2} H_{S T},
\end{aligned}
$$

where $r_{v}$ and $r_{t}$ are the radii of the receiver and transmitter, respectively. In this case, the aperture-averaged logirradiance variance is given by

$$
\begin{aligned}
\sigma_{\ln I, A}^{2}= & 16 \pi^{2} k^{2} L \int_{0}^{1} d u \int_{0}^{\infty} d \kappa \kappa \Phi_{n}(\kappa) \sin ^{2}\left[\frac{\kappa^{2} L u(1-u)}{2 k}\right] \\
& \times\left\{\frac{2 J_{1}\left[\kappa r_{v} u\right]}{\kappa r_{v} u} \frac{2 J_{1}\left[\kappa r_{t}(1-u)\right]}{\kappa r_{t}(1-u)}\right\}^{2} H_{S T}
\end{aligned}
$$

Equation (19) has only a numerical solution. An analytical approximation would simplify the understanding of the basic scintillation phenomena, and would facilitate numerical calculations. To achieve this, the apertures of the transmitter and receiver are modeled using a Gaussian approximation, with PWF and the log-irradiance variance under the Gaussian approximation given by

$$
\begin{aligned}
\operatorname{PWF}_{G}(u)= & 16 \pi^{2} k^{2} L \int_{0}^{\infty} d \kappa \kappa \Phi_{n}(\kappa) \sin ^{2}\left[\frac{\kappa^{2} L u(1-u)}{2 k}\right] \\
& \times \exp \left(-\kappa^{2} r_{v}^{2} u^{2} / 4\right) \exp \left[-\kappa^{2} r_{t}^{2}(1-u)^{2} / 4\right] H_{S T} \\
\sigma_{\ln I, G}^{2}= & 16 \pi^{2} k^{2} L \int_{0}^{1} d u \int_{0}^{\infty} d \kappa \kappa \Phi_{n}(\kappa) \sin ^{2}\left[\frac{\kappa^{2} L u(1-u)}{2 k}\right] \\
& \times \exp \left(-\kappa^{2} r_{v}^{2} u^{2} / 4\right) \exp \left[-\kappa^{2} r_{t}^{2}(1-u)^{2} / 4\right] H_{S T}
\end{aligned}
$$

The Rytov approximation for weak turbulence allows us to set $H_{S T} \approx 1$. Using the Gaussian apertures defined before, the Rytov path-weighting function is now given by

$$
\begin{aligned}
\operatorname{PWF}_{G R}(u)= & 16 \pi^{2} k^{2} L \int_{0}^{\infty} d \kappa \kappa \Phi_{n}(\kappa) \sin ^{2}\left[\frac{\kappa^{2} L u(1-u)}{2 k}\right] \\
& \times \exp \left(-\kappa^{2} r_{v}^{2} u^{2} / 4\right) \exp \left[-\kappa^{2} r_{t}^{2}(1-u)^{2} / 4\right] \\
= & 2.74098 C_{n}^{2} k^{3} R_{f}^{2}\left[r_{v}^{2} u^{2}+r_{t}^{2}(1-u)^{2}\right]^{5 / 6} \\
& \times\left[\left\{1+\frac{16 R_{f}^{4}(1-u)^{2} u^{2}}{\left[r_{v}^{2} u^{2}+r_{t}^{2}(1-u)^{2}\right]^{2}}\right\}^{5 / 12}\right. \\
& \left.\times \cos \left(\frac{5}{6} \arctan \left\{\frac{4\left[R_{f}^{4}(1-u)^{2} u^{2}\right]^{1 / 2}}{r_{v}^{2} u^{2}+r_{t}^{2}(1-u)^{2}}\right\}\right)-1\right] .
\end{aligned}
$$




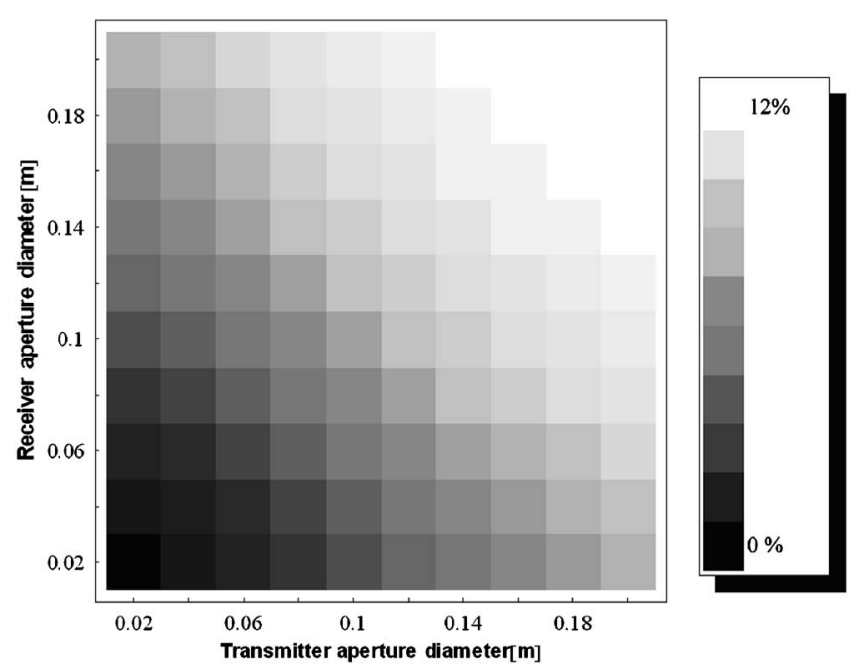

Fig. 1 Systematic relative difference in percent between the Gaussian approximation Eq. (21) and the exact numerical solution of Eq. (19) using the Kolmogorov spectrum and with $H_{S T}=1$. The wavelength used is $4.875 \mu \mathrm{m}$ and the range is $2.5 \mathrm{~km} . C_{n}^{2}=3.4$ $\times 10^{-14} \mathrm{~m}^{-2 / 3}$, and the inner scale $I_{0}=0.005 \mathrm{~m}$. For this situation, the Fresnel range $R_{f}=0.044 \mathrm{~m}$. The horizontal axis is the transmitter aperture diameter, and the vertical axis is receiver aperture diameter.

If the von Karman approximation is used, the following result is obtained for the path-weighting function under the Gaussian aperture assumption, which can be used for computations when the inner scale is of importance:

$$
\begin{aligned}
\operatorname{PWF}_{G K}(u)= & 2.74098 C_{n}^{2} k^{3} R_{f}^{2}\left\{\frac{4+\kappa_{m}^{2}\left[r_{v}^{2} u^{2}+r_{t}^{2}(1-u)^{2}\right]}{\kappa_{m}^{2}}\right\}^{5 / 6} \\
& \times\left[\left(1+\frac{16 \kappa_{m}^{4} R_{f}^{4}(1-u)^{2} u^{2}}{\left\{4+\kappa_{m}^{2}\left[r_{v}^{2} u^{2}+r_{t}^{2}(1-u)^{2}\right]\right\}^{2}}\right)^{5 / 12}\right. \\
& \left.\times \cos \left(\frac{5}{6} \arctan \left\{\frac{4 \kappa_{m}^{2}\left[R_{f}^{4}(1-u)^{2} u^{2}\right]^{1 / 2}}{4+\kappa_{m}^{2}\left(r_{v}^{2} u^{2}+r_{t}^{2}(1-u)^{2}\right)}\right\}\right)-1\right] .
\end{aligned}
$$

Figure 1 shows the systematic relative difference in percent between the Gaussian approximation in Eq. (21) and the exact numerical solution of Eq. (19) using the Kolomogorov spectrum and $H_{S T}=1$. The horizontal axis is the transmitter aperture diameter, and the vertical axis is the receiver aperture diameter. Consistent with our experiments, the wavelength used in the calculations is $4.875 \mu \mathrm{m}$, the range is $2.5 \mathrm{~km}, C_{n}^{2}=3.4 \times 10^{-14} \mathrm{~m}^{-2 / 3}$, and the inner scale $l_{0}=0.005 \mathrm{~m}$. For this situation, the Fresnel range $R_{f}$ $=0.044 \mathrm{~m}$. It can be noted that since both Eqs. (19) and (21) contain the turbulence strength $C_{n}^{2}$ as a multiplicative factor, there is no built-in bias in the Gaussian-aperture approximation for low-turbulence values, compared to the exact calculation.

With the relevant equations available, we can now compare predicted scintillation behavior for several cases of experimental interest. In Fig. 2, we plot the ratio between the log-irradiance variance $\sigma_{\ln I}^{2}$ and the Rytov variance $\beta_{0}^{2}$ as a function of the inner scale radius $l_{0}$. This normalized variance has been computed using both the exact form of Eq. (19) (dashed lines) and the Gaussian approximation of

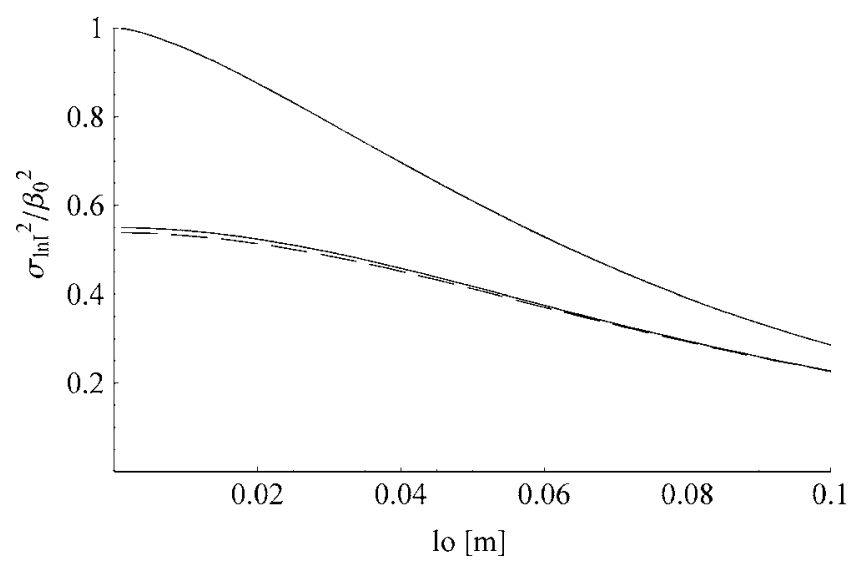

Fig. 2 Rytov-normalized log-irradiance variance as a function of the inner scale radius $I_{0}$, computed using both the exact form of Eq. (19) (dashed lines) and the Gaussian approximation of Eq. (21) (solid lines). Parameters of wavelength, range, turbulence strength, and Fresnel range used for these calculations are the same as in Fig. 1, and $H_{S T}$ has been set to 1 , neglecting saturation effects. Upper curves are for the case of point source and receiver, and lower curves are for the case of the $2.5-\mathrm{cm}$-radius source and receiver.

Eq. (21) (solid lines). Parameters of wavelength, range, turbulence strength, and Fresnel range used for these calculations are the same as in Fig. 1 , and $H_{S T}$ has been set to 1 , neglecting saturation effects. We compare cases for point source and receiver (upper curves), and 2.5-cm-radius source and receiver (lower curves). For the point source/ receiver case, the usual result is obtained, namely that the ratio $\sigma_{\ln I}^{2} / \beta_{0}^{2}$ is unity for zero inner scale. For the finiteapertures case, aperture averaging has decreased the scintillation variance. We see that the Gaussian approximation in Eq. (21) provides very accurate results over a wide range of inner scale radius.

In Fig. 3, we investigate the wavelength dependence of the reduction in Rytov-normalized log-irradiance variance,

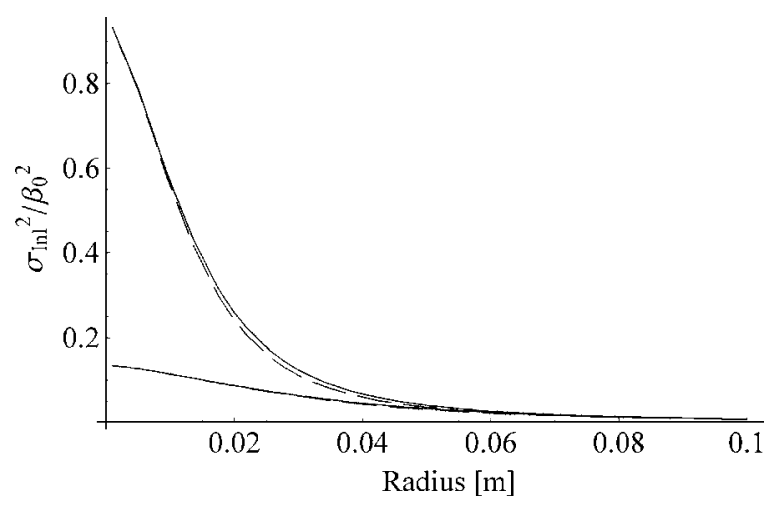

Fig. 3 Rytov-normalized variance normalized log-irradiance variance as a function of equal transmitter and receiver radius. Parameters of wavelength, range, inner scale, and turbulence strength used for these calculations are the same as in Fig. 1, and $H_{S T}$ has been set to 1 , neglecting saturation effects. The upper curves are for $\lambda=880 \mathrm{~nm}$, and the lower curves are for $\lambda=4.875 \mu \mathrm{m}$. The corresponding Fresnel ranges are $R_{f}=0.0187 \mathrm{~m}$ at $\lambda=880 \mathrm{~nm}$ and $R_{f}$ $=0.044 \mathrm{~m}$ at $\lambda=4.875 \mu \mathrm{m}$. As in Fig. 2, the exact calculation from Eq. (19) corresponds to the dashed lines, and the Gaussian approximation of Eq. (21) corresponds to the solid lines. 


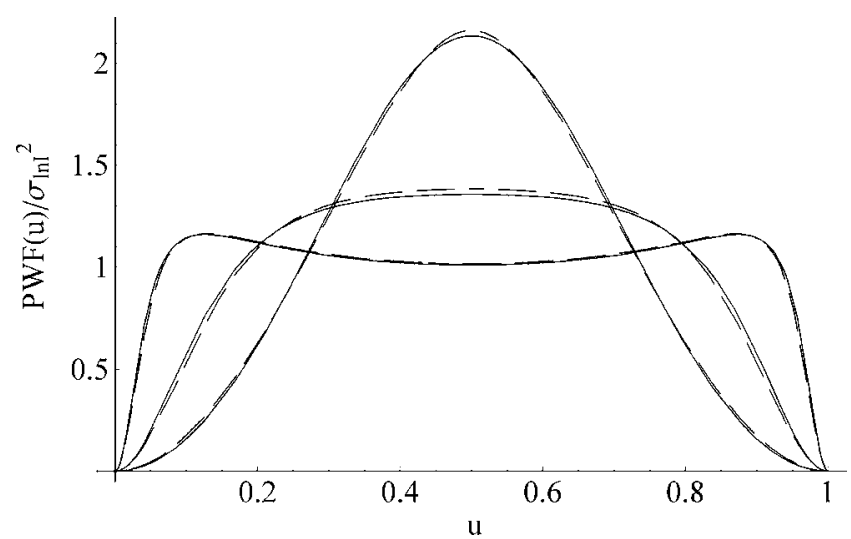

Fig. 4 Variance-normalized path weighting functions versus normalized propagation distance $u$, calculated from the exact expression Eq. (18) (dashed lines) and from the Gaussian expression Eq. (20) (solid lines), using the Kolmogorov spectrum. Curves shown correspond to scattering disk radii of 0,1 , and $4 \mathrm{~m}$ (top to bottom). Saturation effects are included via Eq. (15). The receiver and transmitter radii are $0.2 \mathrm{~m}$.

comparing cases for NIR $\lambda=880 \mathrm{~nm}$ (upper curves) and MWIR $\lambda=4.875 \mu \mathrm{m}$ (lower curves) as a function of equal transmitter and receiver radius. As in Fig. 2, the exact calculation from Eq. (19) corresponds to the dashed lines, and the Gaussian approximation of Eq. (21) corresponds to the solid lines. Parameters of wavelength, range, inner scale, and turbulence strength used for these calculations are the same as in Fig. 1, and $H_{S T}$ has been set to 1 , neglecting saturation effects. The corresponding Fresnel ranges are $R_{f}=0.0187 \mathrm{~m}$ at $\lambda=880 \mathrm{~nm}$, and $R_{f}=0.044 \mathrm{~m}$ at $\lambda$ $=4.875 \mu \mathrm{m}$. The curves show that the wavelength difference in the aperture-averaging effect is significant only for small apertures. Again, the Gaussian-aperture approximation is very accurate over the whole range of radii.

In Figs. 4 and 5, we plot the variance-normalized pathweighting function from the exact expression in Eq. (18) as dashed lines and from the Gaussian expression Eq. (20) as solid lines using the Kolmogorov spectrum as a function of normalized propagation distance $u$ for scattering disk radii of 0,1 , and $4 \mathrm{~m}$. Saturation effects are included via Eq.

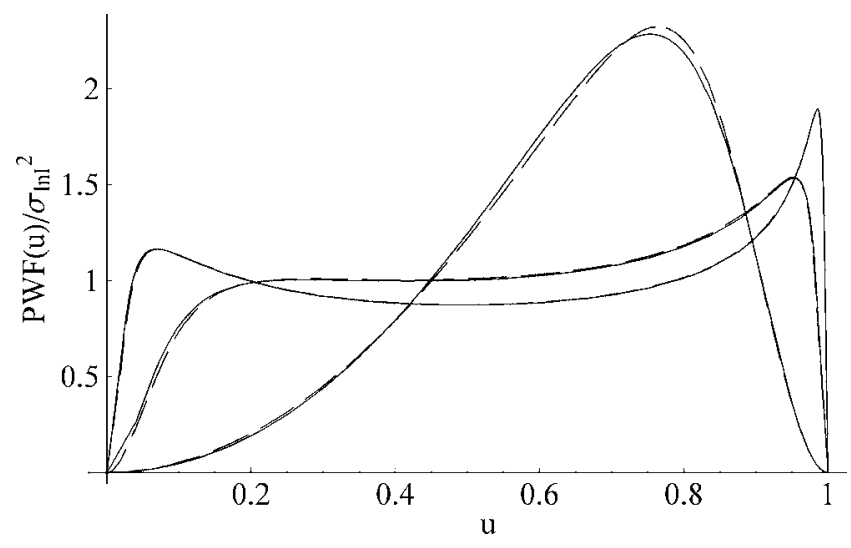

Fig. 5 Variance-normalized path weighting functions as in Fig. 4, except that the receiver and transmitter radii are 0.025 and $0.115 \mathrm{~m}$, respectively, corresponding to our experimental conditions.
(15), since the coherence radius $\rho_{0}$ calculated in Eq. (3) is $0.05 \mathrm{~m}$, large compared to the inner scale of $0.005 \mathrm{~m}$. In Fig. 4, the receiver and transmitter radii are $0.2 \mathrm{~m}$, and in Fig. 5, the receiver and transmitter radii are 0.025 and $0.115 \mathrm{~m}$, respectively, the situation corresponding to our experimental conditions. The Gaussian approximation is seen to have good accuracy in these situations.

\section{Experiments}

We compare scintillation measurements taken with two systems, a commercial NIR scintillometer, and a multispectral MWIR focal plane camera. The scintillometer is a BLS 900, manufactured by Scintec (Longmont, CO). This instrument can provide range-weighted $C_{n}^{2}$ measurements at ranges between 500 and $3000 \mathrm{~m}$, and consists of an optical transmitter, an optical receiver, and a signal-processing unit with software. The transmitter consists of two side-by-side 150-mm-diam circular apertures, each populated with 450 NIR (880-nm) light-emitting diodes (LEDs), and 18 red LEDs for visual alignment. The two transmitters allow estimation of cross-path wind velocity as well as correction of the influence of path attenuation that affects both channels equally. The LED pulse repetition frequency can be varied between 1 and $125 \mathrm{~Hz}$, where higher pulse frequency gives the best measurement accuracy. The narrow field of view (FOV) of the receiver $(8 \mathrm{mrad})$ requires accurate alignment of the instrument. The receiver aperture diameter is $140 \mathrm{~mm}$. The shortest integration time of $1 \mathrm{~min}$ was used for measurement of $C_{n}^{2}$. Parameters available from the scintillometer measurements are the structure parameter $C_{n}^{2}\left(\mathrm{~m}^{-2 / 3}\right)$, Fried's parameter $r_{0}(\mathrm{~m})$; average scintillation index $\sigma_{I}^{2}$, and wind speed $(\mathrm{m} / \mathrm{s})$. The transmitter of the scintillometer was located adjacent to the IR sources, and the receiver was located adjacent to the MWIR camera. Scintillometer measurements were taken immediately before and after each data-acquisition run for the MWIR camera, providing a near-simultaneous measurement of $C_{n}^{2}$ over the propagation path.

The multispectral MWIR imaging sensor is based on a $\mathrm{HgCdTe}(\mathrm{MCT}) 384 \times 288$ pixel focal plane array with sensitivity in the spectral region 1.5 to $5.2 \mu \mathrm{m}$ and a NETD $<25 \mathrm{mK}$. Pixel size is $20 \times 20 \mu \mathrm{m}$ and the distance between pixels is $24 \mu \mathrm{m}$, resulting in $69 \%$ fill factor. Using a $100-\mathrm{mm}$ focal length lens with a $50-\mathrm{mm}$ aperture diameter resulted in a total FOV of $5.3 \times 4.0 \mathrm{deg}$ and a pixel FOV of $0.20 \mathrm{mrad}$. Optical filters were introduced, and the influence of turbulence on received irradiance statistics was measured in three wavebands: band A (2.05 to $2.45 \mu \mathrm{m})$, band $\mathrm{B}$ (3.45 to $4.15 \mu \mathrm{m})$, and band $\mathrm{C}$ (4.55 to $5.2 \mu \mathrm{m})$. Image data were recorded at a rate of 100 frames/sec, with a sensor integration time of $2.6 \mathrm{msec}$. Each data-acquisition run of the MWIR camera was $2 \mathrm{sec}$ in duration. The crosswind speed during the measurements was fairly constant, on the order of $2 \mathrm{~m} / \mathrm{sec}$. The characteristic frequency of the scintillation, as defined by Clifford, ${ }^{11}$ is the cross-wind speed divided by the Fresnel range $R_{f}$. For our situation, this frequency is around $45 \mathrm{~Hz}$. We would thus not expect any appreciable temporal averaging of the scintillation, since the integration time is an order of magnitude shorter than the inverse of the characteristic frequency of the scintillation. 


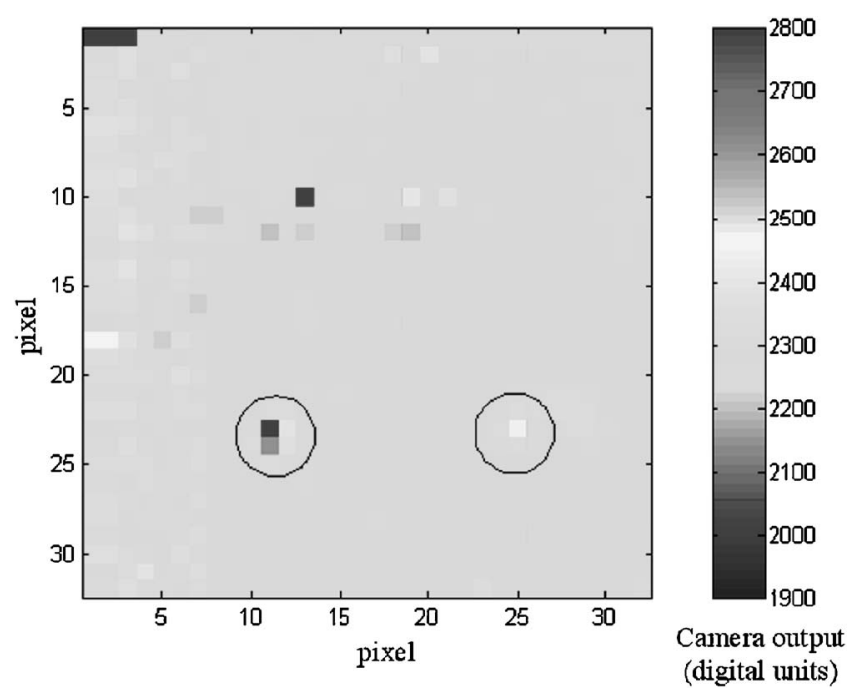

Fig. 6 Instantaneous MWIR camera image in band C showing the hotplate (circled, left-hand side) and the cavity source (circled, righthand side).

Using the MWIR camera, measurements of intensity fluctuations in the three wavelength bands were performed over a $2.5-\mathrm{km}$ range. Two targets were placed within the camera FOV, a cavity blackbody, and a hotplate. The cavity had a 50-mm-diam (essentially a point target at this range with an angular subtense of $20 \mu \mathrm{rad}$ ) and a temperature of $1000 \mathrm{C}$. The hotplate had a $23-\mathrm{cm}$ diameter and a temperature of $350 \mathrm{C}$. Figure 6 shows a dataset in band C containing an instantaneous image of the hotplate and the cavity.

Processing of the datasets proceeded as follows. In each frame of MWIR camera data, the pixel with the highest intensity was identified for both targets, and a $3 \times 3$ pixel region around this peak was summed to yield the level of signal plus local background. The signal level was obtained by subtracting off the local background level, which was determined by averaging the 16 pixels immediately surrounding the $3 \times 3$ pixel region. For any given data point, the log-irradiance variation caused by scintillation was calculated from the 2 -sec time variation of the signal level over the 200-point dataset. Figure 7 shows a representative time history of the signal from the cavity point target, and Fig. 8 shows a typical histogram of irradiance observed. When the histogram is plotted as a function of log irradiance as in Fig. 9, it is seen that the irradiance fluctuations are approximately lognormal.

A summary of the measured data collected is provided in Table 1. The first two data columns show the NIR scintillometer measurements of $C_{n}^{2}$ and the NIR scintillometer measurements of scintillation index $\sigma_{I}^{2}$. Values shown were measured at the beginning and end of each MWIR datacollection run. The next two data columns show the MWIR camera measurements of log-irradiance variance for the hotplate and for the cavity. As expected, the scintillation is more pronounced for the cavity source than for the hotplate.

The data of Table 1 were processed to yield the plots of Figs. 10 and 11. For both figures, the horizontal axis is the $C_{n}^{2}$ measured by the NIR scintillometer (average of readings before and after the corresponding MWIR measurement),

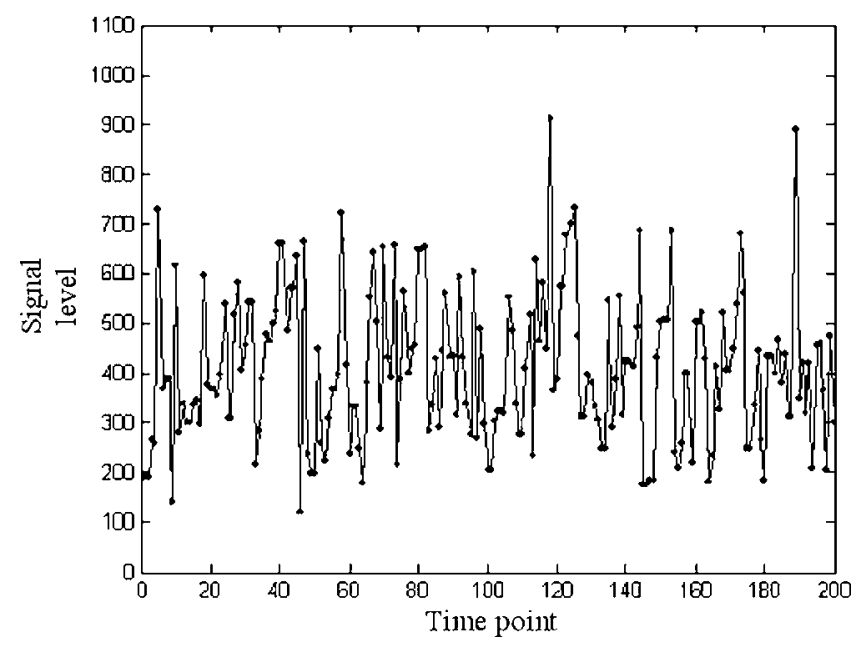

Fig. 7 Typical time history of the signal level from the point target, band $\mathrm{C}$, range $2.5 \mathrm{~km}$.

and the vertical axis is a calculation of $C_{n}^{2}$ values as predicted from the MWIR scintillation-measurement data. Figure 10 uses the Gaussian approximation, Eq. (21), while Fig. 11 uses the exact expression, Eq. (19), with $H_{S T}$ set to unity in both cases. In both cases, measured data values of $\sigma_{\ln I}^{2}$ allow $C_{n}^{2}$ to be predicted, taking the wavelength, path length, and source/receiver aperture dimensions into account. A Tatarskii spectrum was used, with an $l_{0}$ of $5 \mathrm{~mm}$. The results of the calculations were not sensitive to the exact value of $l_{0}$, because of the effects of aperture averaging.

\section{Discussion}

The $C_{n}^{2}$ data of Figs. 10 and 11 are least-squares fitted to a linear dependence, for which the intercept is constrained to zero. This condition is consistent with the earlier observation that both Eqs. (19) and (21) contain the turbulence strength $C_{n}^{2}$ as a multiplicative factor. Any built-in bias in

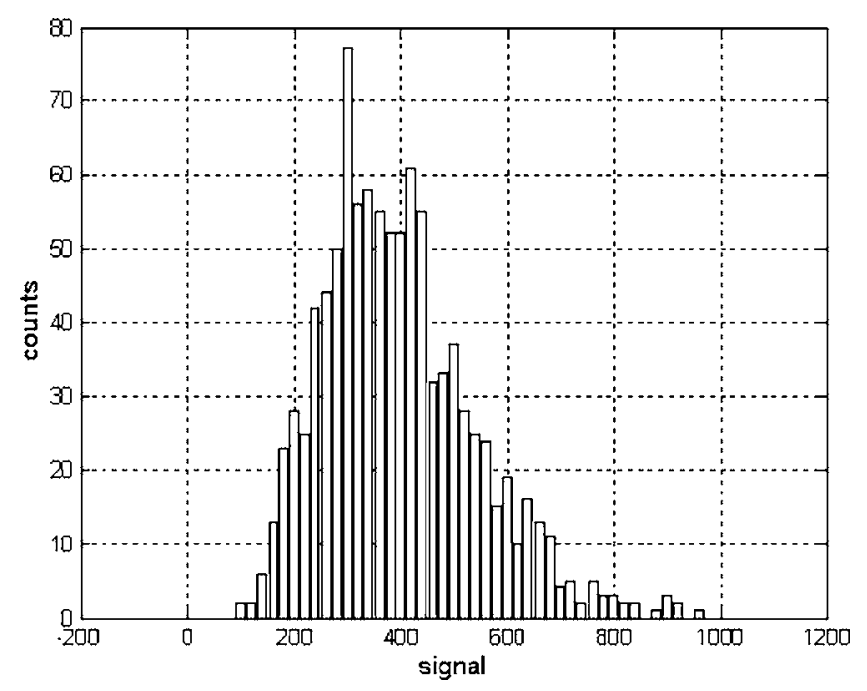

Fig. 8 Typical histogram of signal level from the point target, band $\mathrm{C}$, range $2.5 \mathrm{~km}$. 
Table 1 A summary of the measured data collected. The first two data columns show NIR scintillometer measurements of $C_{n}^{2}$ and scintillation index $\sigma_{l}^{2}$. Values shown were measured at the beginning and end of each MWIR data collection. The next two data columns show MWIR camera measurements of log-irradiance variance for the hotplate and cavity. Filter bands are spectral regions for specific measurements: A (2.05 to $2.45 \mu \mathrm{m}), \mathrm{B}(3.45$ to $4.15 \mu \mathrm{m})$, and C (4.55 to $5.2 \mu \mathrm{m})$.

Measurement 1

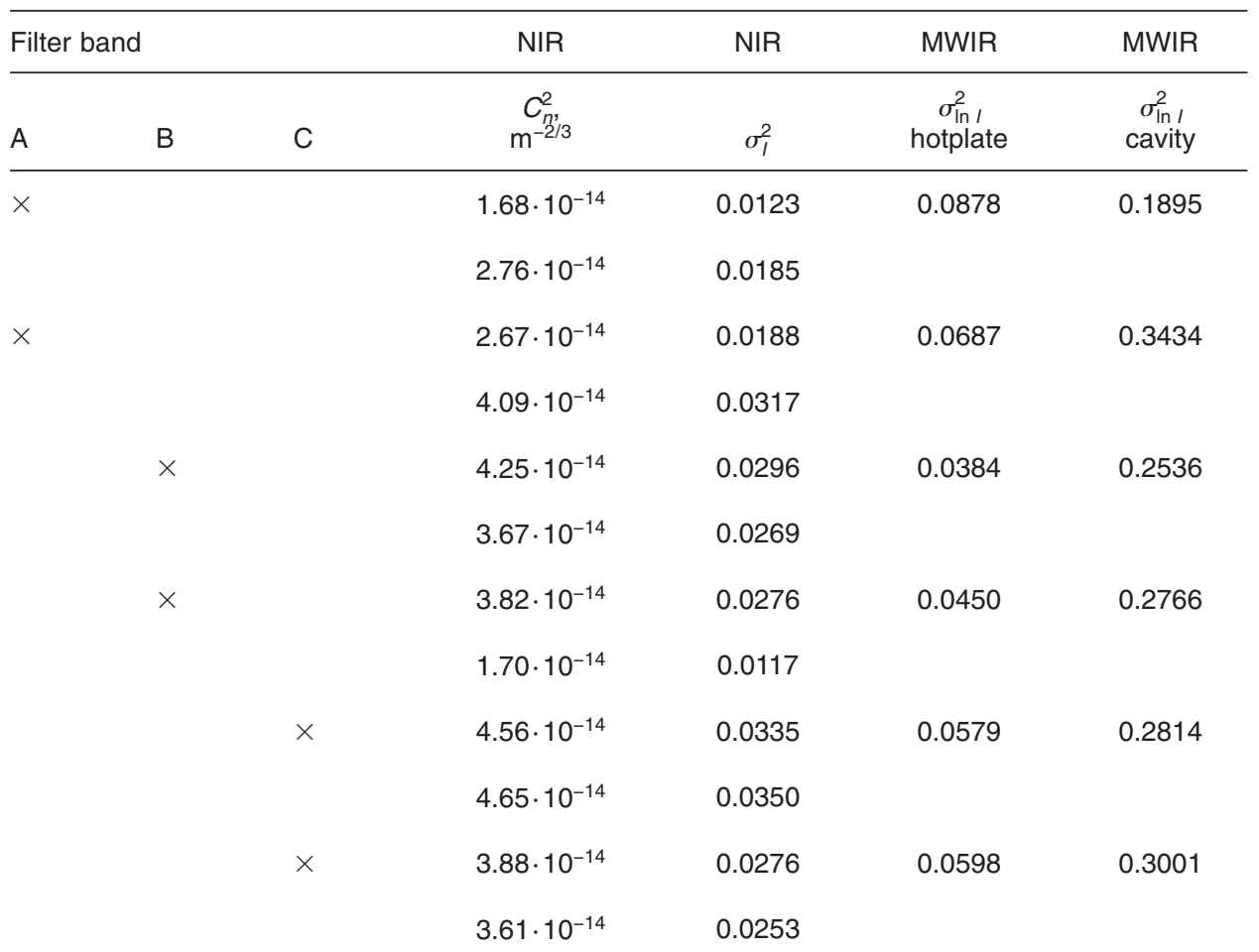

Measurement 2

\begin{tabular}{|c|c|c|c|c|c|c|}
\hline \multicolumn{3}{|c|}{ Filter band } & \multirow{2}{*}{ 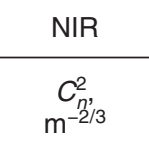 } & \multirow{2}{*}{$\frac{\text { NIR }}{\sigma_{I}^{2}}$} & \multirow{2}{*}{$\frac{\text { MWIR }}{\begin{array}{c}\sigma_{\text {In I }}^{2} \\
\text { hotplate }\end{array}}$} & \multirow{2}{*}{$\begin{array}{c}\text { MWIR } \\
\begin{array}{c}\sigma_{\ln I}^{2} \\
\text { cavity }\end{array}\end{array}$} \\
\hline A & B & C & & & & \\
\hline \multirow[t]{2}{*}{$x$} & & & $2.42 \cdot 10^{-14}$ & 0.0166 & 0.0611 & 0.3453 \\
\hline & & & $2.78 \cdot 10^{-14}$ & 0.0193 & & \\
\hline \multirow[t]{10}{*}{$\times$} & & & $2.78 \cdot 10^{-14}$ & 0.0193 & 0.0598 & 0.3199 \\
\hline & & & $2.80 \cdot 10^{-14}$ & 0.0196 & & \\
\hline & $x$ & & $3.86 \cdot 10^{-14}$ & 0.0286 & 0.0607 & 0.2482 \\
\hline & & & $3.28 \cdot 10^{-14}$ & 0.0231 & & \\
\hline & $x$ & & $3.24 \cdot 10^{-14}$ & 0.0216 & 0.0530 & 0.2092 \\
\hline & & & $3.38 \cdot 10^{-14}$ & 0.0234 & & \\
\hline & & $x$ & $2.89 \cdot 10^{-14}$ & 0.0219 & 0.0399 & 0.1304 \\
\hline & & & $3.95 \cdot 10^{-14}$ & 0.0279 & & \\
\hline & & $x$ & $3.95 \cdot 10^{-14}$ & 0.0272 & 0.0516 & 0.2605 \\
\hline & & & $4.45 \cdot 10^{-14}$ & 0.0317 & & \\
\hline
\end{tabular}




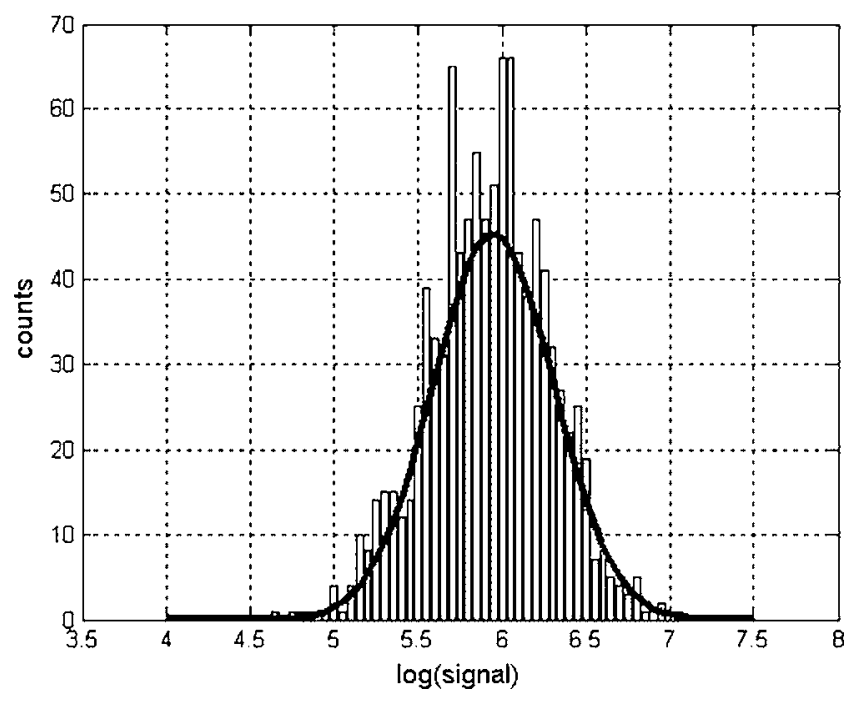

Fig. 9 Typical histogram of the natural log of the signal level observed from the point target, in band $C$, over a range of $2.5 \mathrm{~km}$. Closest-fit lognormal distribution is shown as a solid line.

the calculations would manifest as a nonunit slope in Figs. 10 and 11 , but would not be seen to affect the intercept, since for a situation of $C_{n}^{2}=0$, both equations yield an identically zero value of $\sigma_{\ln I}^{2}$. There is thus no built-in additive bias at low turbulence values in either the Gaussian approximation or the exact calculation. Comparing results for the Gaussian and exact expressions, the least-squares-fit slope was found to be $0.97 \pm 0.06$ in Fig. 10 and $0.97 \pm 0.07$ in Fig. 11. The slope of the ideal curve would be unity. These values show that the Gaussian approximation does not have a built-in multiplicative bias compared to the exact expression, and that NIR scintillometer data for $C_{n}^{2}$ can be used to predict MWIR scintillation levels for two

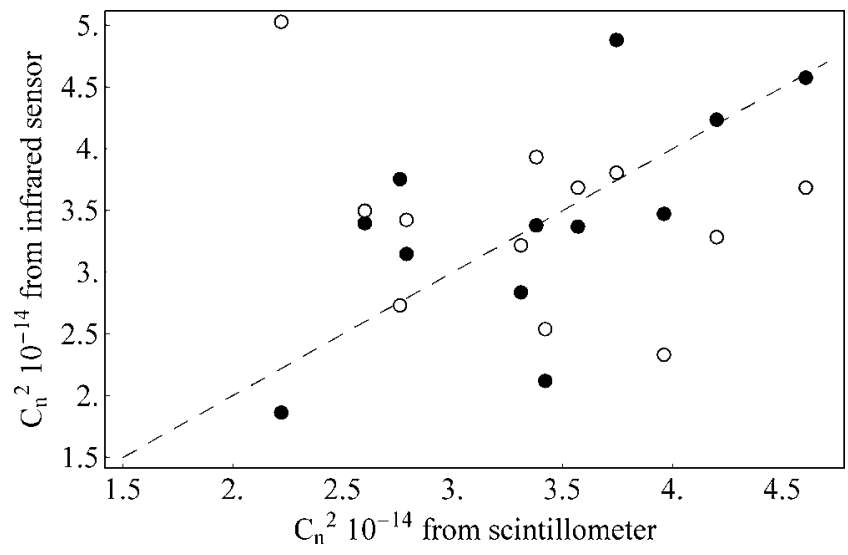

Fig. 10 The horizontal axis is the $C_{n}^{2}$ measured by the NIR scintillometer (average of readings before and after the corresponding MWIR measurement), and the vertical axis is a calculation of $C_{n}^{2}$ values as predicted from the MWIR scintillation-measurement data of Table 1. The Gaussian approximation, Eq. (21), is used, with $H_{S T}$ set to unity. A Tatarskii spectrum was used in the calculation, with an $I_{0}$ of $5 \mathrm{~mm}$. The dashed line is a least-squares linear fit, constrained to have zero intercept. The fitted slope was found to be $0.97 \pm 0.06$, with a correlation coefficient of 0.22 . Filled dots correspond to cavity data, and open dots represent hotplate data.

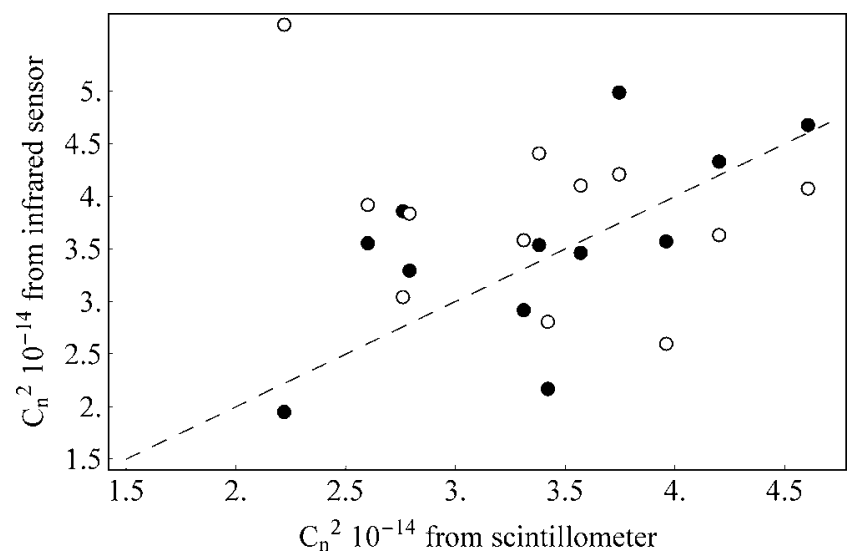

Fig. 11 The horizontal axis is the $C_{n}^{2}$ measured by the NIR scintillometer (average of readings before and after the corresponding MWIR measurement), and the vertical axis is a calculation of $C_{n}^{2}$ values as predicted from the MWIR scintillation-measurement data of Table 1. The exact expression, Eq. (19), is used, with $H_{S T}$ set to unity. A Tatarskii spectrum was used in the calculation, with an $I_{0}$ of $5 \mathrm{~mm}$. The dashed line is a least-squares linear fit, constrained to have zero intercept. The fitted slope was found to be $0.97 \pm 0.07$, with a correlation coefficient of 0.07 . Filled dots correspond to cavity data, and open dots represent hotplate data.

different-sized IR sources at $2.5 \mathrm{~km}$, at least on an average basis. The correlation values of the data are 0.22 for the Gaussian expression used in Fig. 10, and 0.07 for the exact expression used in Fig. 11. These values of correlation show that, for this dataset, it is not possible to establish a closer correspondence of specific measured $C_{n}^{2}$ values between NIR and MWIR data. Investigating possible reasons for the low correlation values, we found that the noise floor of the MWIR camera was not significant on this scale, and also the linearity of the MWIR sensors was excellent over the observed signal range. Surely a more extensive set of experimental studies should be undertaken that spans a larger range of weather conditions, and hence a larger range of $C_{n}^{2}$. However, this half order of magnitude range of $C_{n}^{2}$ was consistent with summer subarctic conditions over a period of several weeks. Comparing scintillation data from bands $\mathrm{A}, \mathrm{B}$, and $\mathrm{C}$, there were no discernable wavelengthdependent trends, nor were source-size trends observed in the residual fitting errors.

Further, it is interesting to note that if saturation is included according to Eq. (15), the calculated MWIR $C_{n}^{2}$ values are consistently high compared to the NIR values, yielding a slope of about 2. Saturation effects will tend to reduce the observed scintillation, and thus higher $C_{n}^{2}$ values are required to be consistent with observed $\sigma_{\ln I}^{2}$ values. In this context, the MWIR scintillations observed in this data were higher than expected, since at a range of $2.5 \mathrm{~km}$, saturation effects would normally have a significant impact.

\section{Conclusions}

Atmospheric turbulence influences received-signal statistics in MWIR small-target-detection scenarios. This will impact both the design of the sensor systems as well as the signal processing. The amount of signal fluctuations can be modeled, taking source and receiver-aperture sizes into account. Expressions are presented for both exact numerical calcu- 
lations and Gaussian-aperture approximations of scintillation. A Gaussian apodization of the source and receiver apertures allows for an accurate analytic solution, with consequent simplifications in calculations and additional insight, compared to the exact equations. This can simplify the performance of parameter-variation trade studies, although the equations are still relatively complex.

Experimental results show that measurements of $C_{n}^{2}$ using a commercial NIR scintillometer allow prediction of average scintillation levels in the MWIR when corrections are made for wavelength and dimensions of the source and receiver. The low correlation values for the datasets indicate that further experimental studies in the MWIR should be carried out, with a goal of increased range of observed $C_{n}^{2}$ values as well as increased volume of data. It is further noted that including saturation effects in the calculations results in overprediction of $C_{n}^{2}$ values by about a factor of 2 . Since saturation would be expected to be significant for a $2.5-\mathrm{km}$ path in the MWIR, the observed values of scintillation in the MWIR are larger than expected, given the NIR measurement data. The received irradiance has an approximate lognormal distribution, which will influence detection probabilities and false-alarm rates for small targets.

\section{References}

1. I. Andrews, R. Phillips, and C. Hopen, Laser Beam Scintillation with Applications, SPIE Press, Bellingham, WA (2001).

2. J. C. Wyngaard, "Atmospheric turbulence," Annu. Rev. Fluid Mech. 24, 205-233 (1992).

3. J. C. Kaimal and J. J. Finnigan, Atmospheric Boundary Layer Flows, Oxford Univ. Press, UK (1994).

4. T. Maurer, R. Driggers, R. Vollmerhausen, and M. Friedman, "2002 NVTherm improvements," Proc. SPIE 4719, 15-23 (2002).

5. L. Andrews, "An analytical model for the refractive index power spectrum and its application to optical scintillations in the atmosphere," J. Mod. Opt. 39, 1849-1853 (1992).

6. L. Andrews and R. Phillips, Laser Beam Propagation Through Random Media, SPIE Press, Bellingham, WA (1998).

7. R. Hill and S. Clifford, "Theory of saturation of optical scintillation by strong turbulence for arbitrary refractive-index spectra," J. Opt. Soc. Am. 71, 675-686 (1981).

8. R. Frehlich and G. Ochs, "Effects of saturation on the optical scintillometer," Appl. Opt. 29, 548-553 (1990).

9. T. Wang, G. Ochs, and S. Clifford, "A saturation-resistant optical scintillometer to measure $C_{n}^{2}$," J. Opt. Soc. Am. 68, 334-338 (1978).

10. R. Hill, "Saturation resistance and inner-scale resistance of a largeaperture scintillometer: a case study," Appl. Opt. 20, 3822-3824 (1981).

11. S. Clifford, "Temporal-frequency spectra for a spherical wave propagating through atmospheric turbulence," J. Opt. Soc. Am. 61, 12851292 (1971).

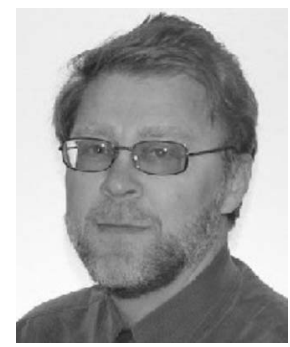

Ingmar G. E. Renhorn received the $\mathrm{PhD}$ degree in physics from the University of Stockholm, Sweden, in 1976. He was a postdoctoral research fellow at the Massachusetts Institute of Technology from 1976 to 1978 . His main research topics were in the combination of conventional diatomicmolecule spectroscopy with laser spectroscopy, as well as hyperfine structure studied by laser and laser-rf techniques. Since 1981, he has been a staff scientist at the Swedish Defense Research Agency (FOI), Linköping, Sweden. In
1994, he was appointed Director of Research at the Department of IR Systems at FOI. His present fields of major interest include multi/ hyperspectral imaging systems, thermal imaging, atmospheric effects on imaging systems, including turbulence and aerosol profiling, imaging theory, image and signal processing, optical information processing, signature measurement, and sensor systems with applications in reconnaissance, infrared search and track, target acquisition, and optical warning.

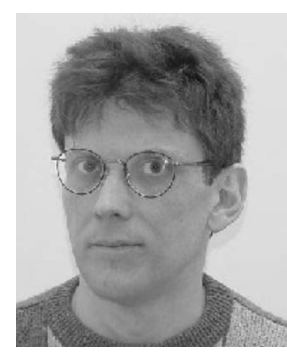

Thomas Svensson received his MSc in chemical engineering at the Lund Institute of Technology in 1996. In 2001, he received his $\mathrm{PhD}$ at Lund University, where his main research was on FTIR spectroscopic studies of molecular complexes of atmospheric interest, using multivariate statistical analysis. He has been employed at FOI since 2001, where his current research interests include applications of infrared sensors.

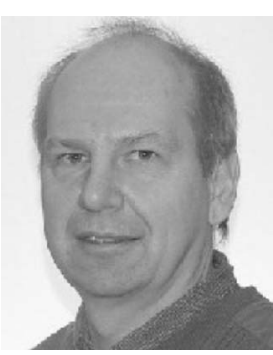

Göran Carlsson studied electrical engineering at Linköping University from 1970 to 1976. He held technical staff positions at Saab and Linköping University Hospital before joining FOI in 1979. His current research interests include applications of infrared sensors.

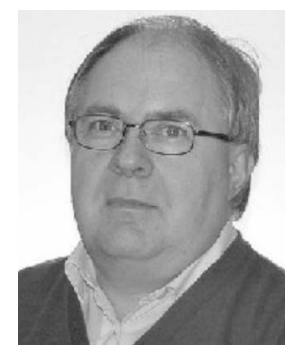

Staffan Cronström received his MSc at the Lund Institute of Technology in 1971. He has held technical staff positions at Linköping Institute of Technology, Context Vision, Innovativ Vision, and AerotechTelub. He has been with FOI since 2000 , where he is a design engineer in the mechanics, electronics, and software areas for the infrared systems department. He has been involved in projects in multiband image acquisition, image fusion, and unmanned air-vehicle

guidance.

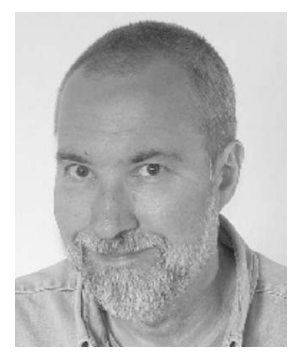

Glenn D. Boreman is Trustee Chair Professor of Optics at University of Central Florida, CREOL. He received a BS in optics from the University of Rochester, and a PhD in optics from the University of Arizona. $\mathrm{He}$ has been a visiting scholar at Imperial College in London, Swiss Federal Institute of Technology (ETH) in Zürich, Complutense University in Madrid, and FOI in Linköping, Sweden. He currently serves as the Editorin-Chief of OSA's journal Applied Optics. He is coauthor of Infrared Detectors and Systems, and author of Modulation Transfer Function in Optical and Electro-Optical Systems, and Basic Electro-Optics for Electrical Engineers. He is a Fellow of SPIE and OSA. 